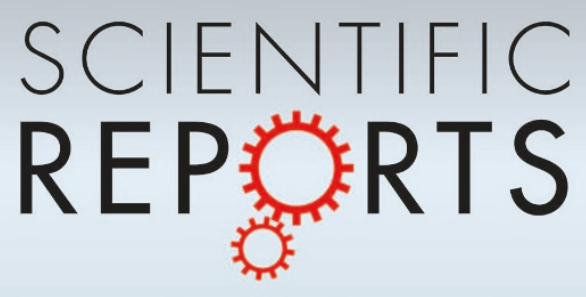

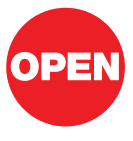

SUBJECT AREAS:

GLASSES

METALS AND ALLOYS

APPLIED PHYSICS

MECHANICAL PROPERTIES

Received

15 November 2012

Accepted

19 December 2012

Published

23 January 2013

Correspondence and requests for materials should be addressed to Z.F.Z. (zhfzhang@imr. ac.cn)

\section{A universal fracture criterion for high-strength materials}

\author{
Rui Tao Qu \& Zhe Feng Zhang
}

Shenyang National Laboratory for Materials Science, Institute of Metal Research, Chinese Academy of Sciences, 72 Wenhua Road, Shenyang, 110016 , P.R. China.

Recently developed advanced high-strength materials like metallic glasses, nanocrystalline metallic materials, and advanced ceramics usually fracture in a catastrophic brittle manner, which makes it quite essential to find a reasonable fracture criterion to predict their brittle failure behaviors. Based on the analysis of substantial experimental observations of fracture behaviors of metallic glasses and other high-strength materials, here we developed a new fracture criterion and proved it effective in predicting the critical fracture conditions under complex stress states. The new criterion is not only a unified one which unifies the three classical failure criteria, i.e., the maximum normal stress criterion, the Tresca criterion and the Mohr-Coulomb criterion, but also a universal criterion which has the ability to describe the fracture mechanisms of a variety of different high-strength materials under various external loading conditions.

$\mathrm{n}$ the $17^{\text {th }}$ century, Galileo Galilei proposed that the fracture of materials often happens at the condition of local normal stress equals to a critical value ${ }^{1,2}$. Now we know that this critical stress condition describes the maximum normal stress criterion, a famous fracture criterion for predicting the brittle fracture of materials ${ }^{3}$. Since then, the efforts to seek for a reasonable and universal fracture criterion have never been stopped because brittle fractures of engineering components are usually disasters causing great loss to human life and property ${ }^{1-3}$. Driving by the urgent requirement of modern industry for high-strength materials, many advanced materials like metallic glasses (MGs), nanocrystalline (NC) metallic materials and advanced ceramics, etc. have been successfully developed and extensively investigated in the past three decades ${ }^{4-7}$. However, one common shortage of these new materials is their brittle fracture behavior or low tensile ductility, which limits their structural applications ${ }^{5,6,8,9}$. The fracture criteria for these high-strength but brittle materials thus become quite essential. Due to the amorphous structure, MGs exhibit not only high strength but also superior formability in the supercooled liquid region, showing promising potential for engineering structural application ${ }^{8,10}$. The high strength, nearly zero tensile ductility, and homogeneous isotropic structure also endow MGs natural model materials to reveal the fracture criterion for those high-strength materials.

The fracture of MG materials usually happens in a shear mode along a very narrow shear band in which severe plastic deformation localizes ${ }^{11,12}$. Besides the shear stress which is the driving force of shear plastic deformation, the normal stress on the shear band plane also influences the fracture behaviors ${ }^{11-16}$. This normal stress effect can be found mainly in three experimental facts ${ }^{11,12,14,17}:$ (1) the fracture strength under uniaxial compression $\sigma_{C}^{F}$ is more or less higher than that under tension $\sigma_{T}^{F}$, showing a tension-compression (T-C) strength asymmetry; (2) the tensile shear band angles $\theta_{T}$ are often much larger than $45^{\circ}$, which is the angle of the maximum resolved shear stress plane; while the compressive shear band angles $\theta_{C}$ are slightly smaller than $45^{\circ}$; (3) the fracture morphologies are also strongly influenced by the normal stress. Therefore, the fracture criterion of MGs should be capable of describing this normal stress effect. For example, the Mohr-Coulomb (M-C) criterion ${ }^{14,15}$, which assumes a linear normal stress dependent shear fracture condition, has been used to explain the shear fracture behaviors of MGs. However, there are several inevitable contradictions between the predictions by the M-C criterion and the experimental results, as elaborated in detail in supplementary materials online (Fig. S1, Table S1). Another fracture criterion, the Ellipse criterion ${ }^{18}$, has been also proposed to interpret the tensile fracture behaviors of MG materials ${ }^{19-21}$. The Ellipse criterion can be expressed as,

$$
\frac{\tau^{2}}{\tau_{0}^{2}}+\frac{\sigma^{2}}{\sigma_{0}^{2}}=1,
$$

where $\tau_{0}$ is the critical shear failure stress, and $\sigma_{0}$ is the critical normal fracture stress, and the ratio $\alpha=\tau_{0} / \sigma_{0}$ is defined as the intrinsic fracture mode factor ${ }^{18,20}$. 
In order to quantitatively examine the validation of the Ellipse criterion, we recently designed some tensile tests on the MG specimens with a series of inclined notches ${ }^{20}$. A wide range of normal stresses acting on the fracture plane was successfully obtained, as shown in Fig. 1. The results show that the critical fracture locus predicted by the Ellipse criterion gives a precise description of the critical stress states of tensile fracture of the studied MG. Furthermore, we found that the Ellipse criterion is capable to describe all the different contributions of normal stress effect on the tensile fracture behavior of a large number of compositionally different $\mathrm{MGs}^{20}$. This suggests that the Ellipse criterion may be suitable as a unified tensile fracture criterion for all MGs.

However, the Ellipse criterion only concerns the cases of tensile stress states, i.e., $\sigma_{1}+\sigma_{3}>0$, where $\sigma_{1}$ and $\sigma_{3}$ are the maximum and minimum principal stresses, respectively. To explain all the fracture behaviors of MGs under various stress conditions, it is necessary to find more general fracture criterion. According to the experimental facts of most MGs as described above, a more general fracture criterion should have the ability to predict: (I) the T-C strength asymmetry, i.e., $\sigma_{C}^{F} / \sigma_{T}^{F} \geq 1$; (II) the shear fracture angles: $0 \leq \theta_{C} \leq 45^{\circ} \leq \theta_{T} \leq 90^{\circ}$; and (III) the critical fracture under pure shear occurring along the plane with the maximum shear stress, i.e., $\left.\frac{d \tau}{d \sigma}\right|_{\sigma=0}=0$. If using the Tresca criterion to predict the compressive fracture behaviors while using the Ellipse criterion to describe the tensile situations ${ }^{21}$, the conditions (I) and (III) are satisfied but the predicted compressive shear fracture angle $\theta_{C}$ will be always equal to $45^{\circ}$. If the fracture behaviors under compressive stress states are described by the $\mathrm{M}-\mathrm{C}$ criterion $^{19}$, then the pure shear fracture cannot be well predicted although the conditions (I) and (II) can be fulfilled. Therefore, this gives rise to a significant scientific question: whether the more general fracture criterion exists or not and it can soundly describe all the fracture behaviors (I)-(III) above?

\section{Results}

Since the Ellipse criterion was experimentally found to be the reasonable expression of the normal stress dependent tensile fracture behaviors, the fracture criterion to describe the fracture behavior of MGs under compressive stress states should possess a similar second-order dependence of shear fracture stress on the normal stress (equation (1)). Note that the fracture behaviors of materials not only depend on the intrinsic factors such as composition and microstructure which can be identified by the intrinsic parameter of

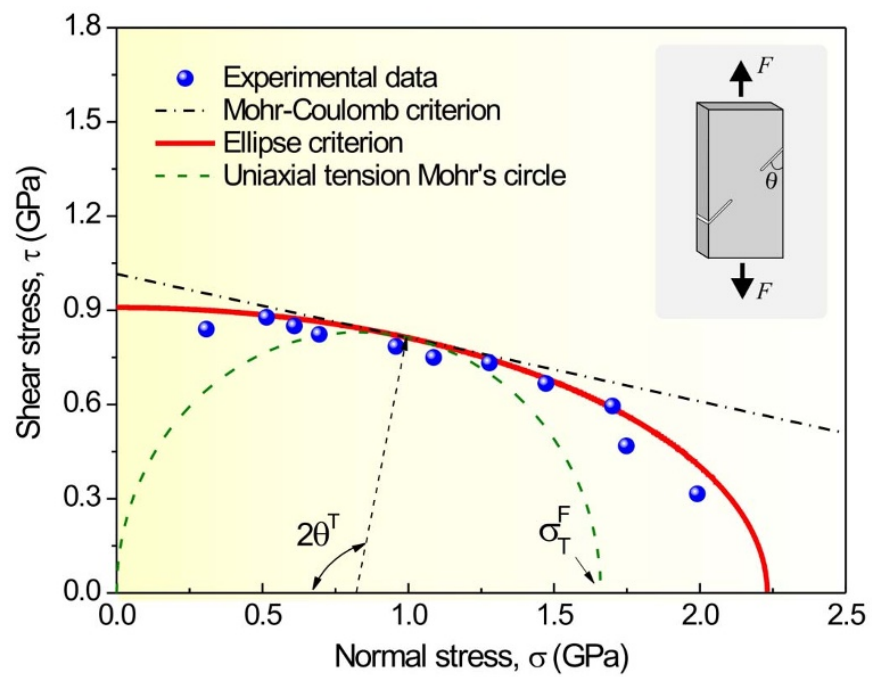

Figure $1 \mid$ Quantitatively experimental verification of the Ellipse

criterion. The experimental data indicates the Ellipse criterion can well predict the tensile fracture behaviors of the $\mathrm{Zr}_{52.5} \mathrm{Cu}_{17.9} \mathrm{Ni}_{14.6} \mathrm{Al}_{10} \mathrm{Ti}_{5} \mathrm{MG}^{20}$. Inset is the sketch of the designed sample. $\alpha=\tau_{0} / \sigma_{0}{ }^{18,20}$, but also influenced by the extrinsic conditions like temperature, strain rate, etc ${ }^{3,11}$. Hence, in order to describe such fracture behaviors dependent on both intrinsic parameters of materials and extrinsic conditions, here we propose a more general fracture criterion which can be written as below,

$$
\tau^{2}+\alpha^{2} \beta \sigma^{2}=\tau_{0}^{2}
$$

$\alpha=\tau_{0} / \sigma_{0}$ is the intrinsic parameter, while $\beta$ is an extrinsic parameter describing the effect of external conditions. At room temperature and quasi-static strain rate, $\beta$ should be different between tensile and compressive stress states because of the following reasons. (1) The directions of normal stresses on the shear fracture plane for tension and compression are always opposite; (2) the compressive normal stress on the fracture behaviors always plays a weaker role in the failure behaviors of materials than the tensile normal stress; one of direct evidences can be found in the experimental fact of the smaller deviation from $45^{\circ}$ of the compressive shear fracture angle than the tensile on $\mathrm{e}^{14}$; (3) the tensile normal stress has much more pronounced effect on the evolution of free volume or shear transformation zone (STZ) and structural defects of MG than the compressive stress ${ }^{13,15,22,23}$. Therefore, for tensile stress states $\left(\sigma_{1}+\sigma_{3} \geq 0\right)$, we suggest that $\beta=\beta_{T}=1$ so that the new criterion has the same form with the original Ellipse criterion of equation (1). For compressive stress states $\left(\sigma_{1}+\sigma_{3}<0\right), \quad \beta=\beta_{C}<0$ and $\left|\beta_{C}\right|<\beta_{T}=1$, which means the compressive normal stress plays a less important role than the tensile one in influencing the critical shear fracture stress. By applying the new criterion to predict the fracture properties under uniaxial tension like tensile fracture strength $\sigma_{T}^{F}$ and tensile fracture angle $\theta_{T}$, the intrinsic factor $\alpha$ and the critical shear fracture strength $\tau_{0}$ can be derived as below:

$$
\begin{gathered}
\alpha=\sqrt{\frac{1}{2}\left(1-\cot ^{2} \theta_{T}\right),} \\
\tau_{0}=\frac{\sigma_{T}^{F}}{2 \sqrt{1-\alpha^{2}}} .
\end{gathered}
$$

By applying the new criterion to predict the compressive fracture angle $\theta_{C}$, the extrinsic parameter under compressive type loading can be further derived as:

$$
\beta_{C}=\frac{1-\cot ^{2} \theta_{C}}{1-\cot ^{2} \theta_{T}} .
$$

According to equations (3)-(5), the three parameters of the new criterion can be achieved through uniaxial tension and compression. In Table 1, we listed the experimental results of the present study and other investigators ${ }^{20,24-28}$ for several $\mathrm{Zr}-$, Ti-, Pd- and Ni-based MGs and also the calculated three parameters of the new criterion. In the present study, the shear fracture angle, which is important for the calculation of $\alpha$ and $\beta_{C}$, were carefully measured and accurately adjusted by considering both the spatial shear plane and elastic recovery ${ }^{20}$ (More details and discussions about the experimental results are shown in supplementary materials). It can be seen from Table 1 that the extrinsic parameter $\beta_{C}$ for the listed MGs at room temperature seems to be always around -0.5 , independent on the composition or microstructure of materials. This conclusion is more affirmative for the present and previous results ${ }^{20}$ in which both the tensile and compressive shear fracture angles were carefully measured. In a microscopic view, the similar value of $\beta_{C}$ for MGs may reflect the similar atomic mechanism of cooperative shearing of STZs for shearing plastic flow ${ }^{13,29}$. The normal stress acting on the shearing plane of STZ influences the behavior of individual STZ and eventually affects the yield and fracture behavior of MGs. The normal stress effect can be specifically described by the new criterion with the intrinsic factor $\alpha$ and the extrinsic parameter $\beta_{C}$. According to the present criterion, $\alpha$ reflects the degrees of normal stress effect, while $\beta_{C}$ describes the differences between the effects by tensile normal 
Table 1 | Experimentally measured fracture strengths $\left(\sigma_{C}^{F}\right.$ and $\left.\sigma_{T}^{F}\right)$, shear fracture angles $\left(\theta_{C}\right.$ and $\left.\theta_{T}\right)$ of different $M G$ materials at room temperature, and the calculated parameters $\left(\alpha, \tau_{0}\right.$ and $\left.\beta_{C}\right)$ of the new criterion

\begin{tabular}{|c|c|c|c|c|c|c|c|c|}
\hline Investigators & Alloys & $\sigma_{C}^{F}(\mathrm{GPa})$ & $\sigma_{T}^{F}(\mathrm{GPa})$ & $\theta_{C}$ (deg.) & $\theta_{T}$ (deg.) & $\tau_{0}(\mathrm{GPa})$ & $\alpha$ & $\beta_{C}$ \\
\hline Present study & $\mathrm{Zr}_{65} \mathrm{Fe}_{5} \mathrm{Al}_{10} \mathrm{Cu}_{20}$ & 1.66 & 1.62 & 42.1 & 53.3 & 0.92 & 0.47 & -0.51 \\
\hline Present study & $\mathrm{Zr}_{50.7} \mathrm{Cu}_{28} \mathrm{Ni}_{9} \mathrm{Al}_{12.3}$ & 1.58 & 1.48 & 42.3 & 53.0 & 0.84 & 0.47 & -0.48 \\
\hline Present study & $\mathrm{Ti}_{40} \mathrm{Zr}_{25} \mathrm{Ni}_{3} \mathrm{Cu}_{12} \mathrm{Be}_{20}$ & 1.84 & 1.68 & 42.6 & 51.8 & 0.93 & 0.44 & -0.47 \\
\hline Present study & $\mathrm{Ti}_{40} \mathrm{Zr}_{25} \mathrm{Ni}_{3} \mathrm{Cu}_{12} \mathrm{Be}_{20}$, annealed & 1.87 & 1.72 & 41.4 & 56.8 & 1.02 & 0.54 & -0.50 \\
\hline Qu et al. ${ }^{20}$ & $\mathrm{Zr}_{52.5} \mathrm{Ni}_{14.6} \mathrm{Al}_{10} \mathrm{Cu}_{17.9} \mathrm{Ti}_{5}$ & 1.84 & 1.66 & 43 & 50.7 & 0.91 & 0.41 & -0.46 \\
\hline Zeng et al. ${ }^{24}$ & $\mathrm{Ni}_{50} \mathrm{Pd}_{30} \mathrm{P}_{20}$ & 1.80 & 1.40 & 41.5 & 55.5 & 0.82 & 0.51 & -0.53 \\
\hline $\begin{array}{l}\text { Caris and } \\
\text { Lewandowski }^{25}\end{array}$ & $\mathrm{Zr}_{41.25} \mathrm{Ti}_{13.75} \mathrm{Ni}_{10} \mathrm{Cu}_{12.5} \mathrm{Be}_{22.5}$ & 2.05 & 1.95 & 42 & 53 & 1.10 & 0.46 & -0.55 \\
\hline Mukai et al. ${ }^{26,27}$ & $\mathrm{Pd}_{40} \mathrm{Ni}_{40} \mathrm{P}_{20}$ & 1.74 & 1.6 & 42 & 56 & 0.94 & 0.52 & -0.43 \\
\hline Zhang et al. ${ }^{28}$ & $\mathrm{Zr}_{52.5} \mathrm{Ni}_{14.6} \mathrm{Al}_{10} \mathrm{Cu}_{17.9} \mathrm{Ti}_{5}$ & 1.76 & 1.66 & 42 & 56 & 0.97 & 0.52 & -0.43 \\
\hline
\end{tabular}

stress and by compressive normal stress. With varying the composition or microstructure, the local properties of STZ (like volume, activation energy etc.) are changed and finally the MG show different tensile yield or fracture behavior ${ }^{29,30}$, which can be well described by the Ellipse criterion with the dominant factor $\alpha$, indicating that $\alpha$ is composition- and microstructure-dependent. However, the similar $\beta_{C}$ for compositionally or structurally different MGs indicates that it should be mainly dominated by extrinsic conditions rather than the intrinsic nature of the materials.

Figure 2 illustrates the graphical representation of the new criterion when applying to a typical MG. A continuous critical fracture locus covers the whole range of normal stress, which allows the prediction of the critical stress conditions for fracture under any stress states. Obviously, the new criterion can well explain all the three characteristic fracture behaviors of MGs, i.e. (I) the T-C strength asymmetry, (II) the shear fracture angles and (III) the pure shear fracture behavior. This indicates that the proposed new criterion satisfies the three necessary conditions for a fracture criterion of MGs.

In order to further examine the rationality of the new criterion and to achieve an overall understanding of the normal stress effect on the fracture behaviors of various MG materials, experimentally measured strengths of more than 30 different compositions of MGs were collected from literatures and listed in Table S3 in supplementary materials. Since the rareness of accurately measured shear fracture angle, the further quantitatively examination of the new criterion will be accomplished by comparing the measured and predicted T-C strength asymmetry. If assuming $\beta_{C}=-0.5$ as obtained in Table 1, according to the new criterion, when the tensile fracture occurs in a shear mode $(0 \leq \alpha \leq \sqrt{2} / 2)$ like in most $\mathrm{MGs}^{14}$, the

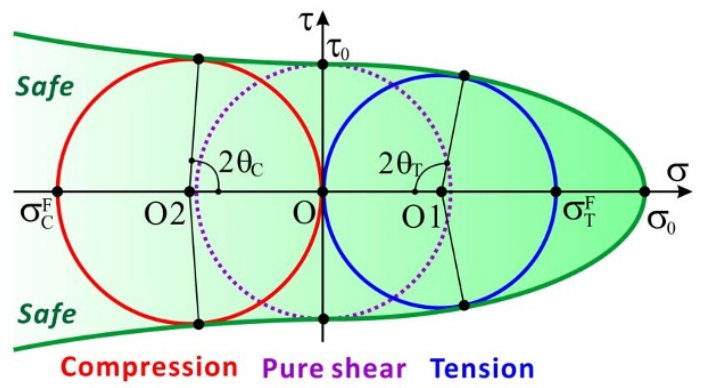

Figure $2 \mid$ Graphical representation of the new fracture criterion. The green line represents the critical fracture locus of the new criterion of equation (2) while the Mohr's stress circles are under uniaxial tension, uniaxial compression and pure shear, respectively. All the three conditions for the fracture criterion describing the fracture behaviors of MGs are obviously fulfilled for the new criterion. The critical fracture locus of the new criterion clarifies that only those stress states enveloped by the fracture locus are safe. fracture strengths $\left(\sigma_{T}^{F}, \sigma_{C}^{F}\right)$ and shear fracture angles $\left(\theta_{T}, \theta_{C}\right)$ under tension and compression can be predicted in terms of the following equations, respectively,

$$
\begin{gathered}
\sigma_{T}^{F}=2 \tau_{0} \sqrt{1-\alpha^{2}}, \\
\sigma_{C}^{F}=2 \tau_{0} \sqrt{1+\alpha^{2} / 2}, \\
\theta_{T}=\operatorname{arccot} \sqrt{1-2 \alpha^{2}}, \\
\theta_{C}=\operatorname{arccot} \sqrt{1+\alpha^{2}} .
\end{gathered}
$$

This will lead to a T-C strength asymmetry with a ratio as below,

$$
\frac{\sigma_{C}^{F}}{\sigma_{T}^{F}}=\sqrt{\frac{1+\alpha^{2} / 2}{1-\alpha^{2}}} \leq \frac{\sqrt{10}}{2} .
$$

When the materials fail in the normal fracture mode under tension as $\alpha>\sqrt{2} / 2$, the tensile fracture strength $\sigma_{T}^{F}$ then becomes the critical cleavage strength $\sigma_{0}$ of the materials, i.e.,

$$
\sigma_{T}^{F}=\sigma_{0}=\tau_{0} / \alpha
$$

And the compression-tension strength ratio becomes,

$$
\frac{\sigma_{C}^{F}}{\sigma_{T}^{F}}=2 \alpha \sqrt{1+\alpha^{2} / 2} .
$$

If the tensile and compressive fracture strengths or their ratios are available, the parameter $\alpha=\tau_{0} / \sigma_{0}$ can be easily calculated in terms of equation (10) or (12). Accordingly, the compression-tension strength ratio $\sigma_{C}^{F} / \sigma_{T}^{F}$ vs. the intrinsic parameter $\alpha=\tau_{0} / \sigma_{0}$ of more than 30 different compositional different MG materials were compared in Table S3 and plotted in Fig. S2 in supplementary materials. It is found that most MGs have the T-C strength asymmetry with a strength ratio $\sigma_{C}^{F} / \sigma_{T}^{F}$ ranging from 1.01 to 1.30 ; and the parameter $\alpha=\tau_{0} / \sigma_{0}$ is thus in the range of $0.1 \leq \alpha \leq 0.6$. According to equations (8) and (9), the tensile and compressive shear fracture angles $\theta_{T}$ and $\theta_{C}$ should be in the ranges of $45^{\circ}<\theta_{T} \leq 62^{\circ}$, and $40^{\circ} \leq \theta_{C}<45^{\circ}$, respectively. These predictions of the shear fracture angles agree well with the reported values in the literatures (e.g., see Table 1 in Ref. 14), further indicating that it is reasonable to quantitatively describe the fracture behaviors of the MG materials with the new fracture criterion.

\section{Discussion}

According to the new criterion, the predictions strongly depend on the intrinsic parameter $\alpha=\tau_{0} / \sigma_{0}$. As illustrated in Fig. 3, with varying $\alpha=\tau_{0} / \sigma_{0}$, three different cases can be classified. When $\alpha=\tau_{0} / \sigma_{0} \rightarrow 0$ or $\sigma_{0} \rightarrow \infty$, the differences in strengths and shear fracture angles under tension and compression can be neglected and both the tensile and compressive shear angles are quite close 
to $45^{\circ}$ (Fig. 3a), which are rather similar to the predictions by the Tresca criterion ${ }^{1,3}$. When $0<\alpha=\tau_{0} / \sigma_{0}<\sqrt{2} / 2$, as shown in Fig. 3b, the compressive fracture strength becomes gradually larger than the tensile one with increasing $\alpha$; meanwhile the compressive shear fracture angle $\theta_{C}$ is smaller than $45^{\circ}$ and the tensile shear fracture angle $\theta_{T}$ is larger than $45^{\circ}$; these approximately agree with the $\mathrm{M}-\mathrm{C}$ criterion. When $\alpha=\tau_{0} / \sigma_{0} \geq \sqrt{2} / 2$, the T-C strength asymmetry becomes much larger and the tensile fracture will always occur along the plane perpendicular to the loading axis, showing the fracture behavior controlled by the maximum normal stress criterion ${ }^{1,18}$. Therefore, the new criterion inherits the unified characteristic of the Ellipse criterion and can be regarded as a criterion unifying the above classical failure criteria.

The above results indicate that the proposed new criterion can not only well predict the fracture conditions of MG materials, but also have the unified characteristic to unify the classical failure criteria. Besides the MG materials, the fracture behaviors of many other highstrength materials including ultrafine-grained (UFG), NC metallic materials and advanced ceramics etc. are influenced more or less by the normal stress acting on the fracture or yield plane $e^{14,31,32}$. For isotropic materials, the T-C strength asymmetry is one of key indicators showing the normal stress effect. A summary of the T-C strength asymmetry for various materials are listed in supplementary Tables S3, S4 and S5 online. As a unified fracture criterion, the new criterion may have the ability to describe the fracture behaviors of a variety of different materials. Although the physical mechanism of the extrinsic parameter $\beta_{C}$ is not very clear for materials other than MG materials, the fracture behaviors including T-C strength asymmetry and fracture mode of various materials can be generally described by the new criterion if assuming $\beta_{C}$ at room temperature and quasi-static strain rate is a constant of -0.5 . Accordingly, the relationship of the material constant $\alpha=\tau_{0} / \sigma_{0}$ versus the T-C strength asymmetry of various different materials can be plotted in Fig. 4, and three regions are classified. For the conventional ductile metallic materials like Al-alloys, Ti-alloys or steels, nearly no difference between tensile and compressive strengths can be observed. The normal stress should only have little influence and the Tresca criterion can be used. The factor $\alpha=\tau_{0} / \sigma_{0}$ is nearly zero and the new criterion in this case is consistent with the Tresca criterion (Fig. 3a). When $\alpha \rightarrow 0$, the normalized strength $\sigma_{T}^{F} / \tau_{0}$ or $\sigma_{C}^{F} / \tau_{0}$ is approximately equal to 2 , which is also the same as the prediction by the Tresca criterion ${ }^{1,3}$. As the grain size of metallic materials decreases into submicrometer or nanometer scales like UFG or NC materials, or MG materials with amorphous structure, the fracture strength increases and the T-C strength asymmetry becomes gradually obvious (region B in Fig. 4). The high strength level and the shear fracture nature of these materials make the normal stress effect hard to be neglected ${ }^{12,13,31,32}$. In addition, different from the metallic materials with metallic bonding, ceramics with either ionic bonding or covalent bonding often display very high strength ratio $\sigma_{C}^{F} / \sigma_{T}^{F}$ even greater than 10 (see supplementary Table S4 online). The strong ionic or covalent bonding greatly increases the resistance to shear deformation and the critical shear failure stress $\tau_{0}$, while the inevitable internal defects like pores decrease the critical normal fracture stress $\sigma_{0}$, leading to the intrinsic factor $\alpha=\tau_{0} / \sigma_{0}$ very large for ceramics. In this case, besides the high $\mathrm{T}$-C strength asymmetry, normal fracture with $\theta_{T}=90^{\circ}$ under tension and shear fracture within $0<\theta_{C}<45^{\circ}$ or even splitting failure ${ }^{14}$ under compression are often observed $^{33}$; these fracture behaviors can be well captured by the present criterion with $\alpha \geq \sqrt{2} / 2$, as illustrated in Fig. 3c.

From the analysis above, it is known that the definition of $\alpha=\tau_{0} / \sigma_{0}$ endows $\alpha$ the materials constant reflecting the intrinsic competition between shear failure and cleavage fracture. Usually low $\alpha$ materials are easy to fail by plastic deformation but hard to cleavage fracture. This is why cleavage fracture is difficult to be observed in ductile FCC crystals like $\mathrm{Cu}$ or $\mathrm{Al}^{3}$. High $\alpha$ materials like ceramics, however, are very easy to cleavage fracture but hard to deform plastically. For materials such as UFG, NC or MG materials, they have moderate $\alpha$ (Fig. 4) and thus often deform and fracture in a shear mode, exhibiting high strength but moderate ductility. Besides the composition and microstructure which identified by the intrinsic factor $\alpha$, the external loading conditions, which can be characterized by the extrinsic parameter $\beta$, also influence the fracture behaviors of materials ${ }^{3}$. For instance, as increasing the testing temperature of nanocrystalline $\mathrm{Al}_{93} \mathrm{Fe}_{3} \mathrm{Cr}_{2} \mathrm{Ti}_{2}$ alloy, the $\mathrm{T}-\mathrm{C}$ strength asymmetry is found to decrease $\mathrm{s}^{34}$. More evidences of the fracture behaviors influenced by the external factors and the interpretations by the present criterion were discussed in supplementary materials. The new criterion suggests that the fracture behaviors are well controlled by both the intrinsic factor $\alpha$ and extrinsic factor $\beta$, which means that even ductile materials with very small $\alpha$ could possibly fracture in a brittle mode under certain external conditions with large $\beta$ and vice versa. These unique characteristics enable the new criterion becoming a fracture criterion not only unifying the classical failure criteria but

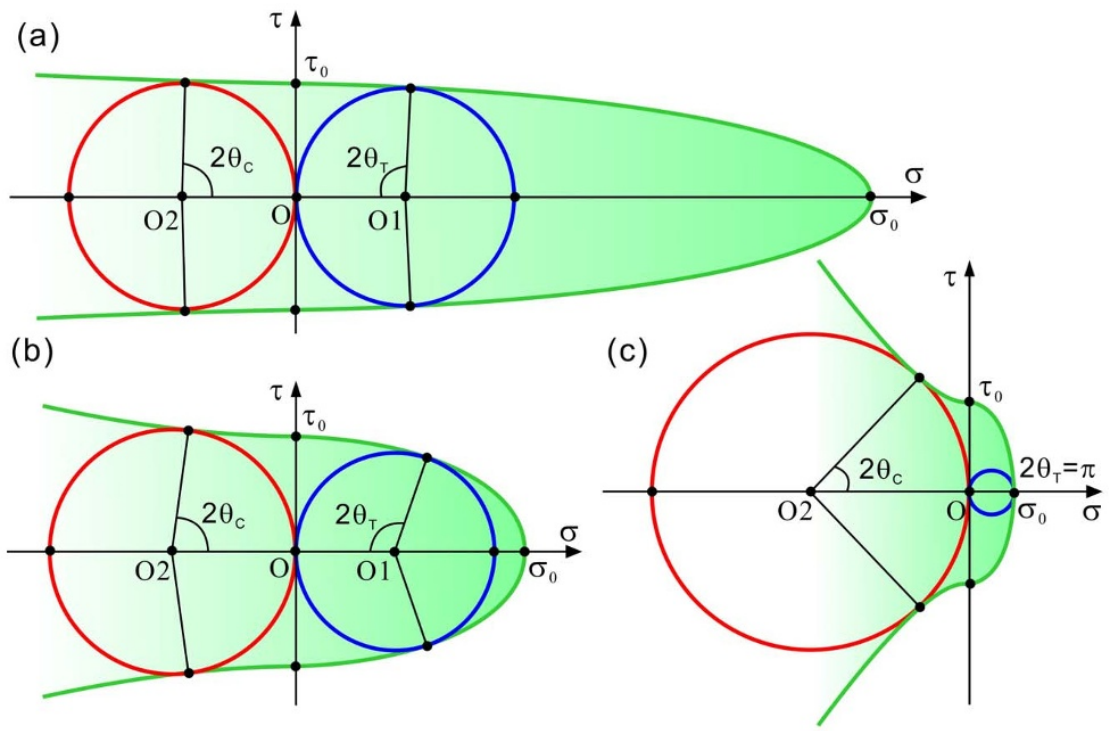

Figure $3 \mid$ Representative critical fracture loci predicted by the new fracture criterion in different cases as varying the intrinsic factor $\alpha=\tau_{0} / \sigma_{0}$. (a) $\alpha \rightarrow 0, \theta_{C} \approx 45^{\circ} \approx \theta_{T}$ and $\sigma_{C}^{F} / \sigma_{T}^{F} \approx 1$; (b) $0<\alpha<\sqrt{2} / 2,0<\theta_{C}<45^{\circ}<\theta_{T}<90^{\circ}$ and $\sigma_{C}^{F} / \sigma_{T}^{F}>1$; (c) $\alpha \geq \sqrt{2} / 2,0<\theta_{C}<45^{\circ}, \theta_{T}=90^{\circ}$ and $\sigma_{C}^{F} / \sigma_{T}^{F} \gg 1$. 


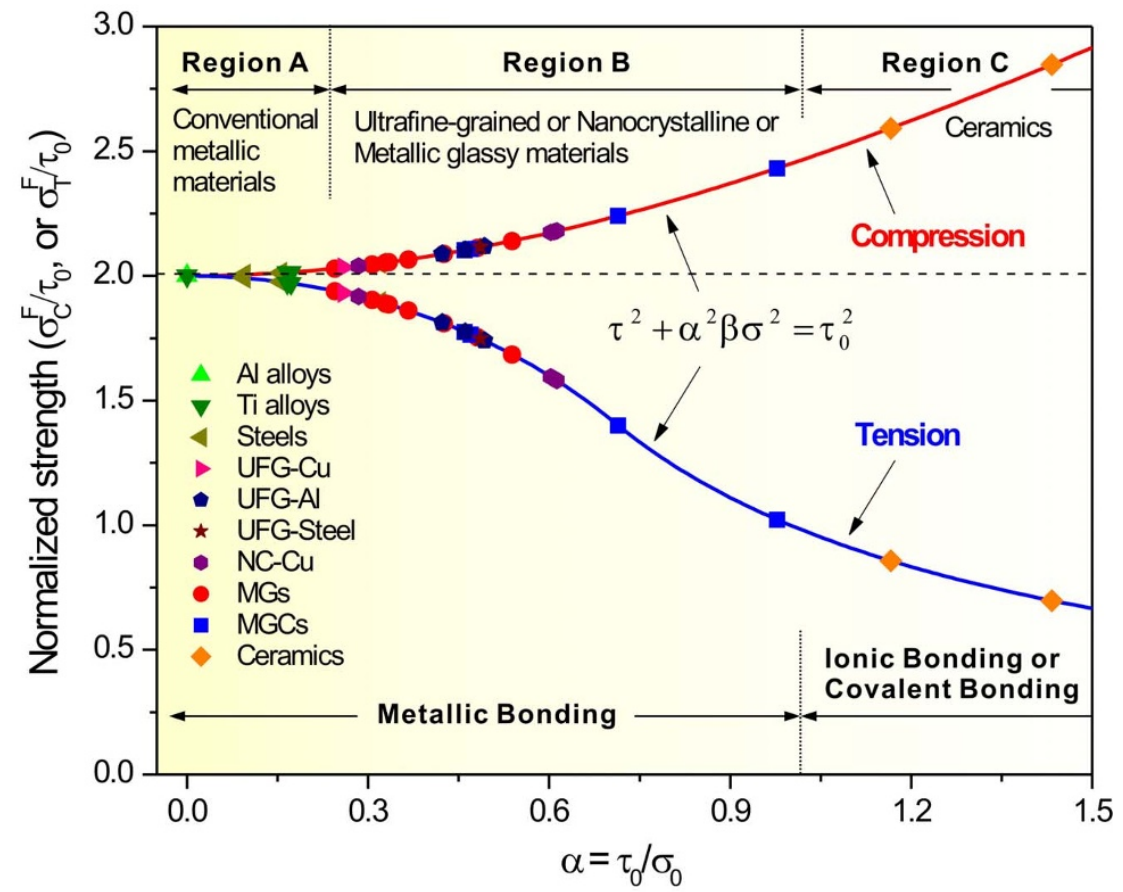

Figure $4 \mid$ Normalized tensile strength $\sigma_{T}^{F} / \tau_{0}$ and normalized compressive strength $\sigma_{C}^{F} / \tau_{0}$ of various different materials (with different $\alpha$ ). The solid lines were drawn according to the new criterion (equation (2)) with $\beta=\beta_{\mathrm{T}}=1$ for tension (blue) and $\beta=\beta_{\mathrm{C}}=-0.5$ for compression (red). Experimental and simulation data of conventional metallic materials, ultrafine-grained (UFG) and nanocrystalline (NC) materials, MGs, MG composites (MGCs) and ceramics were filled based on the data listed in supplementary Tables S3, S4 and S5 online.

also a universal criterion to predict the fracture behaviors of a variety of compositional different materials under various external loading conditions.

In summary, the experimental results of inclined notch tension indicate that the Ellipse criterion can quantitatively predict the tensile fracture behaviors of MGs. In order to cover the whole range of stress states, we extend the Ellipse criterion and propose a new fracture criterion, which not only satisfies the three necessary conditions for fracture criterion of MGs qualitatively but also is able to predict the experimental results quantitatively. Moreover, the new criterion inherits the ability to unify the classical criteria such as the Tresca criterion, the $\mathrm{M}-\mathrm{C}$ criterion and the maximum normal stress criterion in terms of the variation of the intrinsic factor $\alpha=\tau_{0} / \sigma_{0}$. As a result, the new criterion has the ability to well describe the fracture behaviors of various different materials (indentified by the intrinsic factor $\alpha$ ), especially for high-strength materials like UFG, NC, MG materials or advanced ceramics, becoming a universal fracture criterion. As a guide to the engineering design of structural component, the universal criterion gives a continuous critical fracture locus outlining the stress conditions on the most dangerous planes. With the aid of the new fracture criterion on predicting the critical conditions of catastrophic brittle fracture, the structural application of recently developed advanced high-strength materials will become practicable. As a universal fracture criterion, the present criterion also provides a new vision for understanding the fracture mechanisms of various materials in the world.

\section{Methods}

Metallic glasses with nominal compositions of $\mathrm{Zr}_{65} \mathrm{Fe}_{5} \mathrm{Al}_{10} \mathrm{Cu}_{20}, \mathrm{Zr}_{50.7} \mathrm{Cu}_{28} \mathrm{Ni}_{9} \mathrm{Al}_{12.3}$ and $\mathrm{Ti}_{40} \mathrm{Zr}_{25} \mathrm{Ni}_{3} \mathrm{Cu}_{12} \mathrm{Be}_{20}$ were prepared by copper mold casting in a high-purity argon atmosphere. The amorphous structure of the specimens was checked by standard $\mathrm{X}$-ray diffraction. In order to adjust the microstructure intrinsically, some $\mathrm{Ti}_{40} \mathrm{Zr}_{25} \mathrm{Ni}_{3} \mathrm{Cu}_{12} \mathrm{Be}_{20}$ materials were isochronally annealed at $250^{\circ} \mathrm{C}\left(\sim 0.87 T_{\mathrm{g}}\right)$ for 30 minutes. The above four kinds of MG materials, $\mathrm{Zr}_{65} \mathrm{Fe}_{5} \mathrm{Al}_{10} \mathrm{Cu}_{20}$,

$\mathrm{Zr}_{50.7} \mathrm{Cu}_{28} \mathrm{Ni}_{9} \mathrm{Al}_{12.3}, \mathrm{Ti}_{40} \mathrm{Zr}_{25} \mathrm{Ni}_{3} \mathrm{Cu}_{12} \mathrm{Be}_{20}$ and annealed $\mathrm{Ti}_{40} \mathrm{Zr}_{25} \mathrm{Ni}_{3} \mathrm{Cu}_{12} \mathrm{Be}_{20}$, were then used for the subsequent mechanical study. Dog-bone shaped tensile specimens with gauge dimensions of $3 \times 1.5 \times 0.5 \mathrm{~mm}^{3}$ and rectangular compressive specimens with dimensions of $2 \times 2 \times 4 \mathrm{~mm}^{3}$ were cut by electric spark cutting and subsequently grinded and polished by $2.5 \mu \mathrm{m}$ abrasive paste. Uniaxial tension and compression were conducted by using an Instron 5982 testing machine at a nominal strain rate of $5 \times 10^{-4} \mathrm{~s}^{-1}$ at room temperature in air. The deformation and fracture features were observed with a Leo Supra 35 scanning electron microscope.

1. Paul, B. in Fracture, An Advanced Treatise Vol. II (ed Leibowitz, H.) 313-496 (Academic Press, 1968).

2. Yu, M. H. Advances in strength theories for materials under complex stress state in the 20th Century. Appl. Mech. Rev. 55, 169-218 (2002).

3. Meyers, M. A. \& Chawla, K. K. Mechanical Behavior of Materials (Prentice Hall, 1999).

4. Gleiter, H. Nanocrystalline materials. Prog. Mater. Sci. 33, 223-315 (1989).

5. Meyers, M. A., Mishra, A. \& Benson, D. J. Mechanical properties of nanocrystalline materials. Prog. Mater. Sci. 51, 427-556 (2006).

6. Greer, A. L. Metallic glasses. Science 267, 1947 (1995).

7. Barsoum, M. W. \& ElRaghy, T. Synthesis and characterization of a remarkable ceramic: Ti3SiC2. J. Am. Ceram. Soc. 79, 1953-1956 (1996).

8. Ashby, M. F. \& Greer, A. L. Metallic glasses as structural materials. Scripta Mater. 54, 321-326 (2006).

9. Lu, K. The Future of Metals. Science 328, 319-320 (2010).

10. Greer, A. L. Metallic glasses... on the threshold. Mater. Today 12, 14-22 (2009).

11. Schuh, C. A., Hufnagel, T. C. \& Ramamurty, U. Mechanical behavior of amorphous alloys. Acta Mater. 55, 4067-4109 (2007).

12. Zhang, Z. F., Eckert, J. \& Schultz, L. Difference in compressive and tensile fracture mechanisms of $\mathrm{Zr}_{59} \mathrm{Cu}_{20} \mathrm{Al}_{10} \mathrm{Ni}_{8} \mathrm{Ti}_{3}$ bulk metallic glass. Acta Mater. 51, 1167-1179 (2003).

13. Schuh, C. A. \& Lund, A. C. Atomistic basis for the plastic yield criterion of metallic glass. Nature Mater. 2, 449-452 (2003).

14. Zhang, Z. F., He, G., Eckert, J. \& Schultz, L. Fracture mechanisms in bulk metallic glassy materials. Phys. Rev. Lett. 91, 045505 (2003).

15. Donovan, P. E. A yield criterion for $\mathrm{Pd}_{40} \mathrm{Ni}_{40} \mathrm{P}_{20}$ metallic glass. Acta Metall. 37, 445-456 (1989).

16. Murali, P. et al. Atomic Scale Fluctuations Govern Brittle Fracture and Cavitation Behavior in Metallic Glasses. Phys. Rev. Lett. 107 (2011).

17. Qu, R. T., Stoica, M., Eckert, J. \& Zhang, Z. F. Tensile fracture morphologies of bulk metallic glass. J. Appl. Phys. 108, 063509 (2010).

18. Zhang, Z. F. \& Eckert, J. Unified tensile fracture criterion. Phys. Rev. Lett. 94, 094301 (2005).

19. Wu, F. F., Zhang, Z. F., Mao, S. X., Peker, A. \& Eckert, J. Effect of annealing on the mechanical properties and fracture mechanisms of a $\mathrm{Z}_{\mathrm{r} 56.2} \mathrm{Ti}_{13.8} \mathrm{Nb}_{5.0} \mathrm{Cu}_{6.9} \mathrm{Ni}_{5.6} \mathrm{Be}_{12.5}$ bulk-metallic-glass composite. Phys. Rev. B 75 134201 (2007). 
20. Qu, R. T., Eckert, J. \& Zhang, Z. F. Tensile fracture criterion of metallic glass. J. Appl. Phys. 109, 083544 (2011).

21 . Wu, F. F. et al. Strength asymmetry of ductile dendrites reinforced $\mathrm{Zr}-$ and $\mathrm{Ti}-$ based composites. J. Mater. Res. 21, 2331-2336 (2006).

22. Flores, K. M. \& Dauskardt, R. H. Mean stress effects on flow localization and failure in a bullk metallic galss. Acta Mater. 49, 2527-2537 (2001).

23. Jiang, W. H. \& Atzmon, M. The effect of compression and tension on shear-band structure and nanocrystallization in amorphous $\mathrm{Al}_{90} \mathrm{Fe}_{5} \mathrm{Gd}_{5}$ : a high-resolution transmission electron microscopy study. Acta Mater. 51, 4095-4105 (2003).

24. Zeng, Y., Nishiyama, N., Yamamoto, T. \& Inoue, A. Ni-Rich Bulk Metallic Glasses with High Glass-Forming Ability and Good Metallic Properties. Mater. Trans. 50, 2441-2445 (2009).

25. Caris, J. \& Lewandowski, J. J. Pressure effects on metallic glasses. Acta Mater. 58, 1026-1036 (2009).

26. Mukai, T., Nieh, T. G., Kawamura, Y., Inoue, A. \& Higashi, K. Dynamic response of a $\mathrm{Pd}_{40} \mathrm{Ni}_{40} \mathrm{P}_{20}$ bulk metallic glass in tension. Scripta Mater. 46, 43-47 (2002).

27. Mukai, T., Nieh, T. G., Kawamura, Y., Inoue, A. \& Higashi, K. Effect of strain rate on compressive behavior of a Pd40Ni40P20 bulk metallic glass. Intermetallics 10 , 1071-1077 (2002).

28. Zhang, Z. F., Eckert, J. \& Schultz, L. Fatigue and fracture behavior of bulk metallic glass. Metall. Mater. Trans. A 35, 3489-3498 (2004).

29. Johnson, W. L. \& Samwer, K. A Universal Criterion for Plastic Yielding of Metallic Glasses with a $(\mathrm{T} / \mathrm{Tg})^{2 / 3}$ Temperature Dependence. Phys. Rev. Lett. 95, 195501 (2005).

30. Pan, D., Inoue, A., Sakurai, T. \& Chen, M. W. Experimental characterization of shear transformation zones for plastic flow of bulk metallic glasses. Proc. Natl. Acad. Sci. USA. 105, 14769-14772 (2008).

31. Lund, A. C. \& Schuh, C. A. Strength asymmetry in nanocrystalline metals under multiaxial loading. Acta Mater. 53, 3193-3205 (2005).

32. Cheng, S., Spencer, J. A. \& Milligan, W. W. Strength and tension/compression asymmetry in nanostructured and ultrafine-grain metals. Acta Mater. 51, $4505-4518$ (2003).
33. Ramsey, J. M. \& Chester, F. M. Hybrid fracture and the transition from extension fracture to shear fracture. Nature 428, 63-66 (2004).

34. Luo, H., Shaw, L., Zhang, L. C. \& Miracle, D. On tension/compression asymmetry of an extruded nanocrystalline Al-Fe-Cr-Ti alloy. Mater. Sci. Eng. A409, 249-256 (2005).

\section{Acknowledgments}

The authors thank Prof. D. Embury, Prof. M.A. Meyers, Prof. C.A. Schuh and Prof. J. Eckert for helpful and fruitful discussion. This work was financially supported by the National Natural Science Foundation of China (NSFC) under Grant Nos. 50871117 and 50890173, and by the National Basic Research Program of China under Grant No. 2010CB631006.

\section{Author contributions}

Z.F.Z. proposed the idea and designed the research plan. R.T.Q. carried out the experiments and collected the data. Z.F.Z. and R.T.Q. gave the theoretical analysis and wrote the paper.

\section{Additional information}

Supplementary information accompanies this paper at http://www.nature.com/ scientificreports

Competing financial interests: The authors declare no competing financial interests.

License: This work is licensed under a Creative Commons

Attribution-NonCommercial-NoDerivs 3.0 Unported License. To view a copy of this license, visit http://creativecommons.org/licenses/by-nc-nd/3.0/

How to cite this article: Qu, R.T. \& Zhang, Z.F. A universal fracture criterion for high-strength materials. Sci. Rep. 3, 1117; DOI:10.1038/srep01117 (2013). 